\section{Cerebrovascular Diseases}

\section{Introductory Note}

Cerebrovasc Dis 2012;33:97

DOI: $10.1159 / 000334179$

Published online: December 13, 2011

\title{
Methodology, Measures and the Clinician
}

'Look, there was a reduction of 75\%!' This or similar sentences are sometimes addressed to us clinicians, both by colleagues or representatives of drug industries. And we should find enough time to examine in more depth the meaning of the figure which is presented to us. Is it a relative percentage or an absolute one? And is it related to time in some way or just a fixed result? These and other questions we clinicians need to answer when reading papers or listening to presentations of new results from clinical trials are thoroughly addressed in the paper by Knol et al., published in this issue of Cerebrovascular Diseases. In this third paper dealing with methodological and statistical problems, they describe the various measures we frequently read about, comparing their role and specifying when it is sound to use each of them in direct, univariate comparisons. It is particularly important to stress the use of absolute (as opposed to relative) measures when we explain the effect of a treatment to patients, relatives and even to health authorities, but we have also to care- fully filter the information we are continuously fired with and - if necessary - to recalculate the results in terms of absolute differences and number needed to treat. It is also up to us to decide whether it is reasonable to foresee a constant effect over time, or it is perhaps necessary to incorporate some variation of effect in time, when analyzing the results of a trial. Those apparently complex terms like risk and rate ratio, hazard ratio and the like are made understandable, thanks to our Dutch colleagues, and the busy clinician, having read the paper by Knol et al., can now reply to the first sentence: 'OK, but $75 \%$ what?'

We are all aware that there are other problems in interpreting the results of clinical trials: one of the most important is certainly the influence that other variables may have on the effect of the treatment. This problem is usually dealt with by a multivariate (or multivariable) analysis, and this will be the topic of the next paper in the methodological section of Cerebrovascular Diseases.

Stefano Ricci, Città di Castello

\section{KARGER}

Fax +41613061234 E-Mail karger@karger.ch www.karger.com
(C) 2011 S. Karger AG, Basel

1015-9770/12/0332-0097\$38.00/0 